\title{
On the Equivalence of Finite Element and Finite Integration Formulations
}

\author{
A. Demenko ${ }^{1}$, J. K. Sykulski ${ }^{2}$, Fellow, IEEE, and R. Wojciechowski ${ }^{1}$ \\ ${ }^{1}$ Poznan University of Technology, Poznan 60-965, Poland \\ ${ }^{2}$ School of Electronics and Computer Science, University of Southampton, Southampton SO17 1BJ, U.K.
}

\begin{abstract}
The paper offers a comparative study of numerical methods of analysis of electromagnetic fields. The focus is on the finite element method (FEM) and finite integration technique (FIT), but with the cell and equivalent network approaches also considered. It is shown how the approximate integrals describing coefficients of the FEM need to be derived for a mesh with parallelepiped elements to achieve consistency with FIT equations. The equivalence of FEM and FIT formulations for a triangular mesh in 2D is highlighted. The TEAM Workshops Problem No. 7 is used as an example for numerical comparisons. Two formulations have been considered: 1) using the edge values of the magnetic vector potential $A$ and the nodal values of the electric scalar potential $V$; and 2 ) expressed in terms of the edge values of both magnetic $A$ and electric $T-T_{0}$ vector potentials.
\end{abstract}

Index Terms-Eddy currents, electrical engineering education, finite element method (FEM), finite integration technique (FIT), magnetic circuits.

\section{INTRODUCTION}

$\mathbf{T}$ HE finite element method (FEM) has established itself as the prime numerical technique for electromagnetic field computations, but some researchers prefer and promote the use of the finite integration technique (FIT) [1], the cell method (CM) [2], or the equivalent electric and magnetic networks (ENM) [3]. The similarities between CM, FIT, and FEM were observed in [4] and [5], while the analogy between FEM and ENM was explored thoroughly in [6]. The main differences between the different approaches are related to the way in which space is discretized and equation coefficients are set up, in particular the so-called "mass matrices" of the FEM theory [4]. The CM, FIT, and ENM formulations usually rely on a discretization that is equivalent to hexahedral FEM elements of eight nodes and 12 edges (or curved rectangular parallelepipeds under cylindrical symmetry). The FEM mass matrices are nondiagonal, unlike the ones arising in CM, classical FIT, and ENM. The purpose of this paper is to extend the previous comparative analysis of the methods. It is demonstrated that the CM, FIT, and ENM equations may be considered a special case of the FEM formulation. The derived approximate integration formulas yield the equations equivalent (identical).

\section{EQUATIONS OF FEM AND FIT}

Both nodal elements using scalar potentials $\Omega, V$, and edge elements in terms of vector potentials $\boldsymbol{A}, \boldsymbol{T}$ are considered. The FEM equations for scalar potentials correspond to the nodal equations of the edge network with branches coinciding with element edges [6]. The edge model of a rectangular prism is shown in Fig. 1(a). The permeances, conductances, and capacitances forming the mass matrix may be found from

$$
\begin{gathered}
\Lambda_{i, j}^{(p, q)}=\int_{V_{e}} w_{e i, j} \mu \boldsymbol{w}_{e p, q} \mathrm{~d} v \\
G_{i, j}^{(p, q)}+\mathrm{p} C_{i, j}^{(p, q)}=\int_{V_{e}} w_{e i, j}(\sigma+\mathrm{p} \varepsilon) \boldsymbol{w}_{e p, q} d v
\end{gathered}
$$

Manuscript received December 08, 2009; accepted February 10, 2010. Current version published July 21, 2010. Corresponding author: A. Demenko (e-mail: andrzej.demenko@put.poznan.pl).

Color versions of one or more of the figures in this paper are available online at http://ieeexplore.ieee.org.

Digital Object Identifier 10.1109/TMAG.2010.2043506

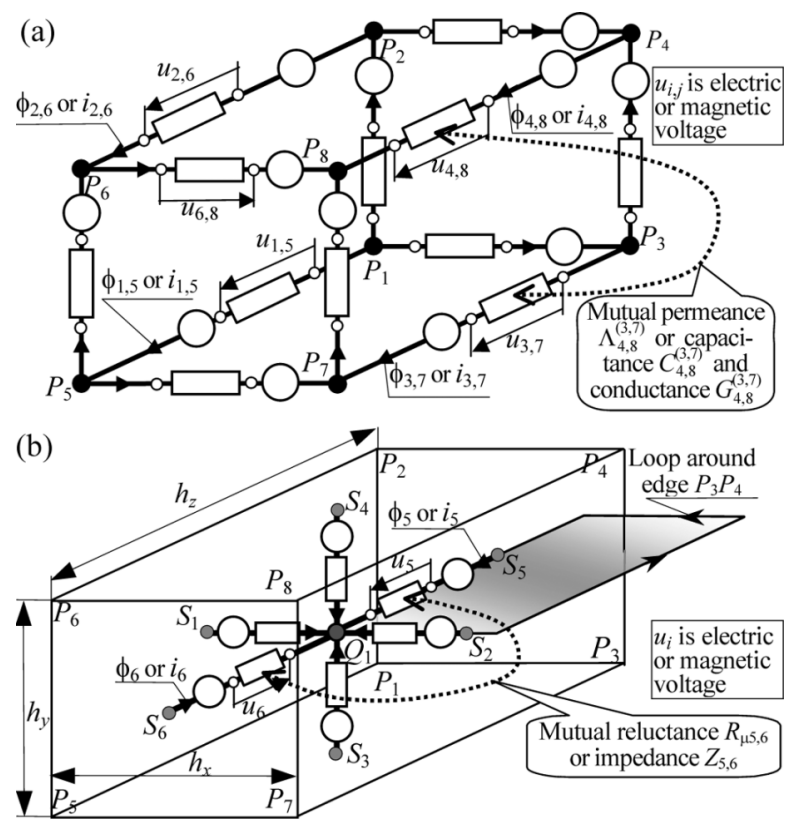

Fig. 1. (a) Edge and (b) facet model of an element.

where $\boldsymbol{w}_{e i, j}, \boldsymbol{w}_{e p, q}$ are interpolation functions of an edge element for the edges $P_{i} P_{j}$ and $P_{p} P_{q}$, respectively; $\mathrm{p}=\mathrm{d} / \mathrm{d} t$; and $V_{e}$ is the volume of the element. The FEM equations for vector potentials and edge elements represent loop equations of the facet network, the branches of which cross the element facets.

A portion of a network of a parallelepiped element is shown in Fig. 1(b). The reluctances and impedances of the element model relate to the mass matrix elements and are described by

$$
\begin{aligned}
R_{\mu i, q} & =\int_{V_{e}} \boldsymbol{w}_{f i} \mu^{-1} \boldsymbol{w}_{f q} d v \\
Z_{i, q} & =\int_{V_{e}} \boldsymbol{w}_{f i}(\sigma+\mathrm{p} \varepsilon)^{-1} \boldsymbol{w}_{f q} \mathrm{~d} v
\end{aligned}
$$

where $\boldsymbol{w}_{f i}, \boldsymbol{w}_{f q}$ are interpolation functions of a facet element for the facets $S_{i}, S_{q}$ [6].

The FEM mass matrices are nondiagonal; consequently, so are the matrices of the equivalent network models. In the models 


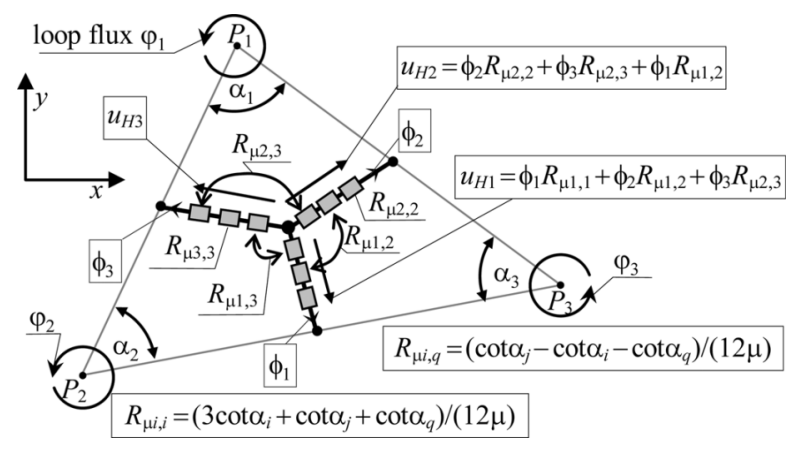

Fig. 2. Reluctance (facet) model of a triangle with mutual reluctances.

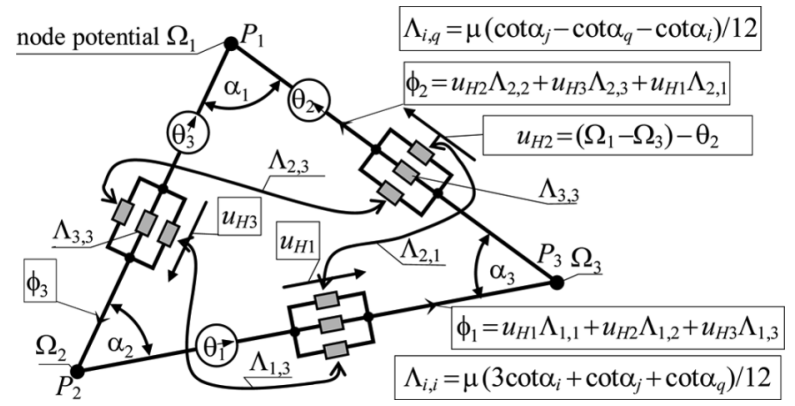

Fig. 3. Permeance (facet) model of a triangle with mutual permeances.

of Fig. 1, the branches that are not perpendicular to each other will have a mutual coupling. Both the flux and the current in branch $P_{i} P_{j}$ of the edge model depend on voltages in the other branches parallel to $P_{i} P_{j}$, for example,

$$
i_{1,5}=\sum_{j=1}^{4}\left(G_{j, j+4}^{(1,5)}+\mathrm{p} C_{j, j+4}^{(1,5)}\right) u_{j, j+4} .
$$

In the above relationship, the conductances and capacitances are defined as follows:

$$
\begin{aligned}
& G_{1,5}^{(1,5)}=2 G_{2,6}^{(1,5)}=2 G_{3,7}^{(1,5)}=4 G_{4,8}^{(1,5)}=\sigma h_{y} h_{x} /\left(9 h_{z}\right) \\
& C_{1,5}^{(1,5)}=2 C_{2,6}^{(1,5)}=2 C_{3,7}^{(1,5)}=4 C_{4,8}^{(1,5)}=\varepsilon h_{y} h_{x} /\left(9 h_{z}\right)
\end{aligned}
$$

where $h_{y}, h_{x}$ and $h_{z}$ are dimensions as in Fig. 1(b).

In the facet model of Fig. 1(b), the electric/magnetic potential differences across the branch $Q_{1} S_{i}$ depend on the current/flux in the branches parallel to $Q_{1} S_{i}$; for example, the "magnetic voltage" (that is a magnetic potential difference across the reluctance) $u_{5}$ of branch $Q_{1} S_{5}$ may be written as

$$
u_{5}=R_{\mu 5,5} \phi_{5}+R_{\mu 5,6} \phi_{6}
$$

where

$$
R_{\mu 5,5}=-2 R_{\mu 5,6}=h_{z} /\left(3 \mu h_{x} h_{y}\right) .
$$

Coupling will also occur within the facet and edge models of a triangular element; see Figs. 2 and 3. Fig. 2 shows a reluctance (facet) model of a triangular element that has been derived using a facet model of a five-sided prism [7]. Here, the loop fluxes $\varphi_{i}$ are given by the product of the $A_{z}$ component of the potential $A$ and the length $l(l=1 \mathrm{~m})$ in the $z$-direction. The edge (permeance) model of a triangular element, i.e., the $2 \mathrm{D}$ model for a

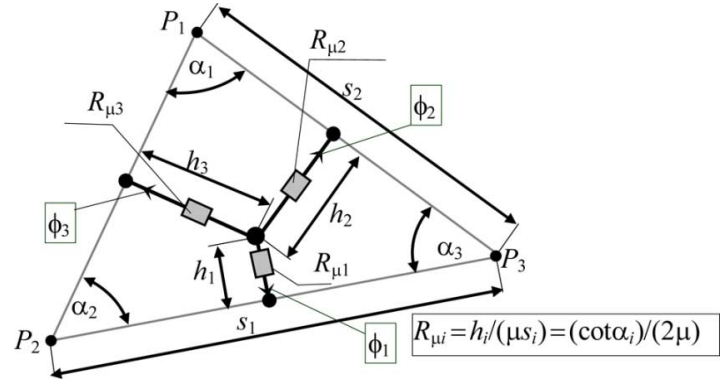

Fig. 4. Reluctance (facet) model of a triangle without mutual reluctances.

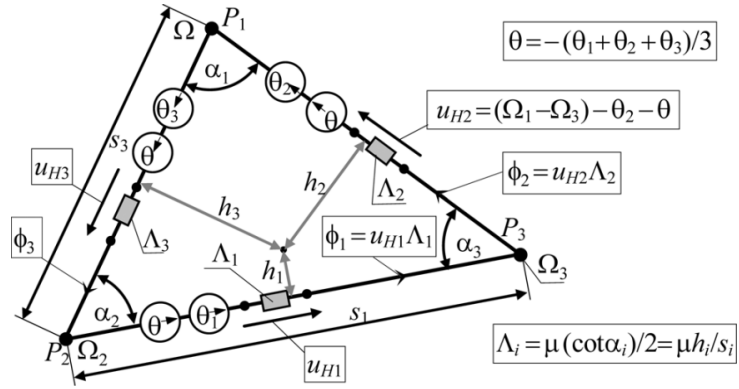

Fig. 5. Permeance (facet) model of a triangle without mutual permeances.

formulation using the scalar potential $\Omega$, is shown in Fig. 3. In the model, the branch magnetomotive forces (mmfs) $\theta_{i}$ represent edge values of $\boldsymbol{T}$ or $\boldsymbol{T}_{0}$, i.e., $\theta_{1}$ is the edge value of $\boldsymbol{T}$ or $\boldsymbol{T}_{0}$ for $P_{2} P_{3}$.

Equations arising from the CM, classical FIT, and ENM formulations may appear to be similar to those obtained from the FEM, but there is an important difference: They do not contain mutual couplings, and thus the mass matrices are diagonal. For example, for the models of Fig. 1, we can write

$$
\begin{aligned}
R_{\mu 5,5} & =R_{\mu 6,6}=h_{z} /\left(2 \mu h_{x} h_{y}\right) \\
\Lambda_{i, i+4}^{(i, i+4)} & =\mu h_{x} h_{y} /\left(4 h_{z}\right),(i=1,2,3,4) .
\end{aligned}
$$

In the reluctance and permeance models of a triangle

$$
\begin{aligned}
R_{\mu i, i} & =R_{\mu i}=h_{i} /\left(\mu s_{i}\right) \\
\Lambda_{i, i} & =\Lambda_{i}=\mu h_{i} / s_{i}
\end{aligned}
$$

with $h_{i}$ and $s_{i}$ as shown in Figs. 4 and 5.

\section{DERIVING FEM EQUIVALENT TO FIT}

From circuit theory, it is well known that a three-branch star with mutual couplings may be converted into an equivalent one without any couplings, as demonstrated by Fig. 4. This may be achieved by exploiting the condition $\Sigma \phi_{i}=0$, which allows the expressions for the "magnetic voltage" in the branch with fluxes $\phi_{1}, \phi_{2}$ (see Fig. 2) to be written as

$$
\begin{aligned}
u_{H 1}-u_{H 2}= & R_{\mu 1,1} \phi_{1}+R_{\mu 1,2} \phi_{2}+R_{\mu 1,3} \phi_{3}-R_{\mu 2,2} \phi_{2} \\
& -R_{\mu 2,1} \phi_{1}-R_{\mu 2,3} \phi_{3}=R_{\mu 1} \phi_{1}-R_{\mu 2} \phi_{2}
\end{aligned}
$$

where the reluctances $R_{\mu i}(i=1,2)$ are described in Fig. 4. As a result, a model may be derived containing no mutual reluctances but having a diagonal mass matrix with self-reluctances identical to the FIT formulation (Fig. 4). 
The mutual couplings may also be eliminated from the edge model of Fig. 3. When formulating the nodal equations and finding the fluxes "entering" the nodes, account needs to be taken of the condition $\Sigma\left(u_{H i}+\theta_{i}\right)=0$ applicable to the triangular loop. Consequently, using the notation from Fig. 3, the flux entering node $P_{1}$ equals

$$
\phi_{2}-\phi_{3}=\Lambda_{2}\left(\Omega_{3}-\Omega_{1}-\theta_{2}-\theta\right)-\Lambda_{3}\left(\Omega_{2}-\Omega_{1}-\theta_{3}-\theta\right)
$$

where the $\operatorname{mmf} \theta$ and the permeances $\Lambda_{i}(i=2,32)$ are depicted in Fig. 5.

For the 2D models considered, it is therefore possible to start with different mass matrices for FEM and FIT and yet achieve identical matrix of coefficients for both formulations. The above transpositions, regrettably, do not apply to 3D systems. In 3D cases, it is possible to derive FEM equations equivalent to FIT formulation and arising from the integrals (1) and (2), describing the elements of the mass matrix, by applying the following approximation

$$
\int_{V_{e}} f \mathrm{~d} v=\frac{V_{e}}{n_{n}} \sum_{i=1}^{n_{n}} f\left(P_{i}\right)
$$

where $n_{n}$ is the number of element nodes $P_{i}$, and $f\left(P_{i}\right)$ is the value of the function $f$ in the node $P_{i}$.

This formula may result in models free of mutual couplings, thus with coefficients the same as if obtained from FIT. Unfortunately, the procedure described by (11) is only successful-in terms of making the matrix diagonal-in the case of parallelepiped elements (it also works for curved rectangular parallelepipeds), i.e., for elements with $n_{n}=8$. The mass matrices of tetrahedral and five-sided prism elements may be made diagonal only if complemented by additional assumptions regarding fluxes or currents-for example, by imposing (or assuming) one of the facet flux or current densities in a tetrahedron to be negligibly small.

Interesting results are obtained when (11) is applied to a fivesided element (Fig. 6) in that no coupling will occur between parallel branches, e.g., between $P_{1} P_{4}, P_{3} P_{6}$ and $P_{1} P_{3}, P_{4} P_{6}$ in the edge model or between $Q S_{4}$ and $Q S_{5}$ in the facet model. Both FIT and FEM formulations using (11) lead to the same description of those elements of the mass matrix that represent the parameters of the branch $P_{i} P_{i+3}$ in the edge model, for example $\Lambda_{z i, j}=0$ (for $i \neq j$ ) and $\Lambda_{z i, i}=\mu S /(3 h)$, where $S$ is the area of the triangle face and $h$ is the element height (Fig. 6). The coefficients related to the parameters of the branch $Q S_{4}$, $Q S_{5}$ in the facet model are also identical, e.g., $R_{\mu 4,5}=0$ and $R_{\mu 4,4}=R_{\mu 5,5}=h /(2 S)$. However, despite using (11) in both formulations, the FEM and FIT result in different descriptions of such entries in the mass matrix that are related to the branches $P_{i} P_{j},(i, j=1,2,3 ; i \neq j), P_{p} P_{q},(p, q=4,5,6 ; p \neq q)$ in the edge model and the branches $Q S_{i}(i=1,2,3)$ in the facet model. Using FEM and (11) gives

$$
\begin{aligned}
\Lambda_{i, j} & =\frac{\mu h}{12} \cot \alpha_{q} \\
\Lambda_{i, i} & =\frac{\mu h}{12}\left(2 \cot \alpha_{i}+\cot \alpha_{j}+\cot \alpha_{q}\right) \\
R_{\mu i, j} & =\frac{1}{6 \mu h} \cot \alpha_{q} \\
R_{\mu i, i} & =\frac{1}{6 \mu h}\left(2 \cot \alpha_{i}+\cot \alpha_{j}+\cot \alpha_{q}\right)
\end{aligned}
$$

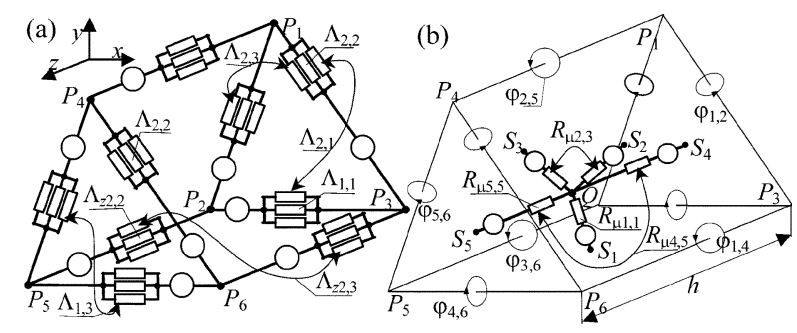

Fig. 6. Circuit models of a five-sided element: (a) edge; (b) facet.

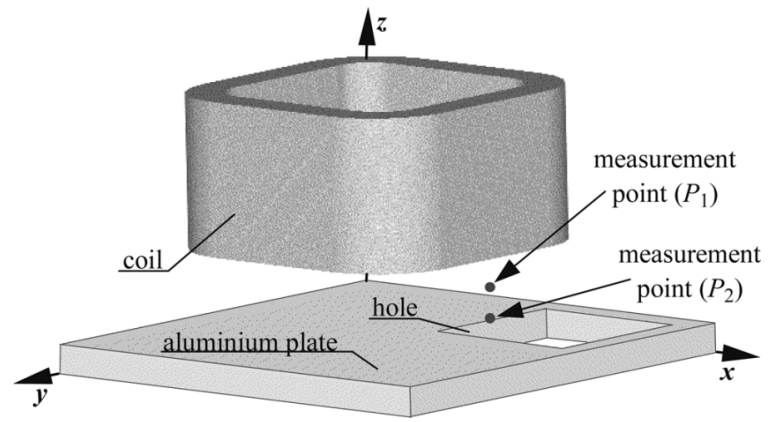

Fig. 7. TEAM Workshops Problem No. 7.

where $\alpha_{i}, \alpha_{j}, \alpha_{q}$ denote angles related to nodes $P_{i}, P_{j} . P_{q}$ of the triangular "base" as shown in Fig. 3.

The derived edge model containing couplings between branches may be converted into a model without couplings where $\Lambda_{i, j}=0$. To achieve this, similar transformations are needed as in the case of the $2 \mathrm{D}$ edge models described by Figs. 3 and 5. It may be shown that

$$
\begin{aligned}
\Lambda_{i, j} & =0 \\
\Lambda_{i, i} & =0.25 \mu h \cot \alpha_{i} .
\end{aligned}
$$

Unfortunately, as in the 2D case, the transformations change the description of the sources; an additional $\operatorname{mmf} \theta$ will appear in the converted network (Fig. 5).

The facet model of the element obtained from FEM and (11) — as described by (13) - may be converted into a coupling-free model following an assumption that the sum of the fluxes in branches $Q S_{4}$ and $Q S_{5}$ is negligibly small, thus-as in the 2D case-we have $\phi_{1}+\phi_{2}+\phi_{3}=0$. After some further manipulations, we find

$$
\begin{aligned}
R_{\mu i, j} & =0 \\
R_{\mu i, i} & =0.5(\mu h)^{-1} \cot \alpha_{i} .
\end{aligned}
$$

As a result of these transformations, the loop mmf does not change, thus the right-hand side vector of the FEM equations is not affected.

\section{EXAMPLE}

The TEAM Workshops Problem No. 7 (Fig. 7) has been selected to illustrate the theoretical investigations [8], [9]. The magnetic field and eddy current distributions have been calculated for a conducting plate with a hole, with the excitation provided by a multiturn coil.

Two formulations have been adopted: 1) an $\boldsymbol{A}-\boldsymbol{V}$ formulation with edge values of magnetic vector potential $\boldsymbol{A}$ and nodal values of electric scalar potential $V$; and 2) an $\boldsymbol{A}-\boldsymbol{T}-\boldsymbol{T}_{0}$ method with edge values of magnetic $\boldsymbol{A}$ and electric $\boldsymbol{T}-\boldsymbol{T}_{0}$ vector potentials [10]. The bounded space has been subdivided into about 
TABLE I

COMPARISON BETWEEN FIT AND FEM RESULTS FOR $\boldsymbol{A}-\boldsymbol{V}$ FORMULATION

\begin{tabular}{|c|c|c|}
\hline Method & FIT & FEM \\
\hline \multicolumn{3}{|c|}{ Flux density in point $\boldsymbol{P}_{\mathbf{1}}$} \\
\hline $\boldsymbol{B}_{\boldsymbol{x}}[\mathbf{T}]$ & -0.010689 & -0.010747 \\
\hline $\boldsymbol{B}_{\boldsymbol{y}}[\mathbf{T}]$ & 0.003581 & 0.003583 \\
\hline $\boldsymbol{B}_{z}[\mathbf{T}]$ & 0.008145 & 0.008165 \\
\hline \multicolumn{3}{|c|}{ Current density in point $\boldsymbol{P}_{\mathbf{2}}$} \\
\hline $\boldsymbol{J}_{\boldsymbol{x}}\left[\mathbf{A} / \mathbf{m}^{\mathbf{2}}\right]$ & 83275.85 & 83196.15 \\
\hline $\boldsymbol{J}_{\boldsymbol{y}}\left[\mathbf{A} / \mathbf{m}^{\mathbf{2}}\right]$ & 1713894.52 & 1710454.18 \\
\hline $\boldsymbol{J}_{z}\left[\mathbf{A} / \mathbf{m}^{\mathbf{2}}\right]$ & -39703.18 & -39469.81 \\
\hline
\end{tabular}

TABLE II

COMPARISON BETWEen FIT AND FEM RESUlts For $\boldsymbol{A}-\boldsymbol{T}-\boldsymbol{T}_{0}$ Formulation

\begin{tabular}{|c|c|c|}
\hline Method & FIT & FEM \\
\hline \multicolumn{3}{|c|}{ Flux density in point $\boldsymbol{P}_{\mathbf{1}}$} \\
\hline $\boldsymbol{B}_{\boldsymbol{x}}[\mathbf{T}]$ & -0.010476 & -0.010495 \\
\hline $\boldsymbol{B}_{\boldsymbol{y}}[\mathbf{T}]$ & 0.003482 & 0.003512 \\
\hline $\boldsymbol{B}_{z}[\mathbf{T}]$ & 0.008058 & 0.008063 \\
\hline \multicolumn{3}{|c|}{ Current density in point $\boldsymbol{P}_{\mathbf{2}}$} \\
\hline $\boldsymbol{J}_{\boldsymbol{x}}\left[\mathbf{A} / \mathbf{m}^{2}\right]$ & 81927.33 & 81634.45 \\
\hline $\boldsymbol{J}_{\boldsymbol{y}}\left[\mathbf{A} / \mathbf{m}^{2}\right]$ & 1629026.65 & 1628932.24 \\
\hline $\boldsymbol{J}_{z}\left[\mathbf{A} / \mathbf{m}^{2}\right]$ & -38420.30 & -38384.68 \\
\hline
\end{tabular}

150000 elements, some 16000 of which were placed in the conducting region.

First, consider the results of an $\boldsymbol{A}-V$ formulation. The resultant system of equations corresponds to a reluctance-conductance network consisting of about half a million loop equations related to the magnetic network and 20000 nodal equations of the electric network. The relevant parameters for the FEM model were derived using (1) and (2), thus creating mutual conductances and reluctances. A block relaxation method, combined with incomplete Cholesky decomposition, has been used to solve the final system of equations. Table I shows example values of the flux and current densities at points $P_{1}$ and $P_{2}$ as marked in Fig. 7. The selection of these points was guided by available TEAM results [9], [11], as the published comparisons refer to flux densities in $P_{1}$ and current densities in $P_{2}$. The values are given for an instant of time when the coil current was at its maximum (a $50-\mathrm{Hz}$ supply has been assumed).

A formulation in terms of $\boldsymbol{A}-\boldsymbol{T}-\boldsymbol{T}_{0}$ was also considered. The relevant equations are in fact loop equations of the magnetic and electric networks. In the example, the number of loop equations of the electric network was 63000 , while the number of equations of the magnetic network was the same as in the $A-V$ approach. The relevant parameters for the model were derived using (2) for the FEM, thus creating mutual resistances and reluctances. For the FIT formulation, the coefficients were calculated using (2), where the integrals were approximated using (11). The resulting equations were solved using a block relaxations method in a similar way as for the $\boldsymbol{A}-V$ solution. The computed flux densities for $P_{1}$ and current densities for $P_{2}$ are summarized in Table II.

The current densities presented in both Tables I and II are close to values published in [9] and [11]. Moreover, similar discrepancies to those reported and highlighted in [9] have been observed in relation to the values obtained using the potential $V$ against the results based on $\boldsymbol{T}-\boldsymbol{T}_{0}$.

For all points considered, the differences between the FIT and FEM results do not exceed $0.6 \%$ for flux density and $0.7 \%$ for current density. It therefore appears reasonable to conclude that the proposed approximation (11) — which leads to equations equivalent to the FIT method with a diagonal mass matrix-is perfectly acceptable without noticeable loss of accuracy. Moreover, the diagonal matrix is easy to invert, thus seeking edge values of $A$, representing loop fluxes in the model of Fig. 1(b), may be conveniently replaced by an easier task of finding nodal potentials associated with element centers (nodes $Q_{i}$ ). In the case of diffusion problems, the additional advantage of making the mass matrix diagonal is a possibility of applying explicit numerical schemes [4].

\section{CONCLUSION}

The FEM and FIT formulations have been compared, with particular emphasis on the analysis of the mass matrix. The differences between the formulations were explained using the "language" of circuit theory. In the circuit equivalents of the FEM method, there are couplings between branches, whereas such couplings are absent from the FIT description. It was demonstrated that, in the 2D case, the coupled models may be transformed into coupling-free equivalents. In the 3D formulation, the FIT-equivalent FEM models are possible only under special circumstances; the elements of the mass matrix must be computed using a suggested approximation (11). The reported test results have confirmed that the differences as a consequence of accurate or approximate integral computations are small. Thus, in order to benefit from the computational advantages of the diagonalization of the FIT matrix, it is recommended to use the proposed approximation (11). For the parallelepiped elements, this reduces the problem of solving the FEM equations to a simpler and more efficient solution of FIT equations.

\section{REFERENCES}

[1] T. Weiland, "A discretization method for the solution of Maxwell's equations for six-component fields," Electron. Commun. AE ̈̈, vol. 31, no. 3, pp. 116-120, 1977.

[2] E. Tonti, "A direct formulation of field laws: The cell method," Comput. Model. Eng. Sci., vol. 2, no. 2, pp. 237-258, 2001.

[3] J. Sykulski et al., Computational Magnetics. London, U.K.: Chapman \& Hall, 1995.

[4] A. Bossavit and L. Kettunen, "Yee-like schemes on staggered cellular grids: A synthesis between FIT and FEM approaches," IEEE Trans. Magn., vol. 36, no. 4, pt. 1, pp. 861-867, Jul. 2000.

[5] M. Clemens, S. Feigh, and T. Weiland, "Construction principles of multigrid smoothers for curl-curl equations," IEEE Trans. Magn., vol. 41, no. 5, pp. 1680-1683, May 2005.

[6] A. Demenko and J. K. Sykulski, "Network equivalents of nodal and edge elements in electromagnetics," IEEE Trans. Magn., vol. 38, no. 2, pt. 1, pp. 1305-1308, Mar. 2002.

[7] A. Demenko, L. Nowak, and W. Szelagg, "Reluctance network formed by means of edge element method," IEEE Trans. Magn., vol. 34, no. 5, pt. 1, pp. 2485-2488, Sep. 1998.

[8] L. Turner, Ed., "TEAM Workshops: Test problems No. 7," in Proc. Workshops Problems Benchmarking Eddy Current Codes, Argonne, IL, Apr. 1988, pp. 19-26.

[9] O. Biro, K. Preis, W. Renhart, K. R. Richter, and G. Vrisk, "Performance of different vector potential formulations in solving multiply connected 3D eddy current problems," IEEE Trans. Magn., vol. 26, no. 2, pp. 438-441, Mar. 1990.

[10] A. Demenko, J. Sykulski, and R. Wojciechowski, "Calculation of inducted currents using edge elements and $\boldsymbol{T}-\boldsymbol{T}_{0}$ formulation," Sci., Meas. Technol., vol. 2, no. 6, pp. 434-439, Nov. 2008.

[11] W. Rucker and K. Richter, "A BEM code for 3-D eddy current calculations," IEEE Trans. Magn., vol. 26, no. 2, pp. 462-465, Mar. 1990. 